\title{
Pathogenesis of Atopic Dermatitis and Psoriasis: Focus on the Epidermal Differentiation Complex
}

\author{
Judith G.M. Bergboer, Patrick L.J.M. Zeeuwen and Joost Schalkwijk*
}

\author{
Department of Dermatology, Radboud University Nijmegen Medical Centre, Nijmegen Centre for Molecular Life \\ Sciences, Nijmegen, The Netherlands
}

\begin{abstract}
Loss-of-function mutations in the $F L G$ gene (encoding the epidermis-specific protein filaggrin) were recently shown to be associated with atopic dermatitis in several populations. These findings have challenged the dogma that atopic dermatitis is primarily an immune-mediated disease, and suggest skin barrier deficiency as a major cause. Similarly, psoriasis was until recently regarded as a T-cell driven disease caused by (auto)immune mechanisms. This view is supported by clinical data, and several genetic studies have identified risk factors associated with a function in adaptive immunity such as HLA-Cw6. Analysis of the PSORS4 region on chromosome 1q, however, has recently identified the deletion of late cornified envelope $(L C E)$ genes $L C E 3 B / C$ as novel psoriasis genes with a considerable population attributable risk. Because these genes are expressed in epithelial cells and not by the immunocytes, these findings have changed our view on psoriasis. The mechanism by which loss of $L C E 3 B / C$ genes predisposes to psoriasis is unclear as both genes are not expressed in normal skin but appear to be induced upon skin barrier disruption or inflammation. We hypothesize that psoriasis could be the result of an interplay of immunological mechanisms and deficient repair of skin barrier integrity. Altogether, these findings demonstrate that in addition to immune mechanisms, genetic variation of skin barrier genes contribute to major skin diseases such as atopic dermatitis and psoriasis.
\end{abstract}

Keywords: Atopic dermatitis, epidermal differentiation complex, filaggrin, late cornified envelope, $L C E 3 B / C$ deletion, psoriasis.

\section{GENETIC STUDIES ON SKIN DISEASES: INVOLVE- MENT OF THE EPIDERMAL DIFFERENTIATION COMPLEX}

Atopic dermatitis and psoriasis are clinically distinct, chronic inflammatory skin diseases with a strong genetic basis, and are extensively reviewed [1-5]. Several genomewide analyses have been performed to discover genetic factors contributing to atopic dermatitis and psoriasis. These analyses have revealed chromosomal regions harboring possible susceptibility loci for both diseases on chromosomal regions $1 \mathrm{q} 21,3 \mathrm{q} 21,17 \mathrm{q} 25$ and $20 \mathrm{p} 12$. In this review we will focus on the overlapping locus on $1 \mathrm{q} 21$, for psoriasis the socalled psoriasis susceptibility (PSORS) 4 locus. This shared locus overlies the epidermal differentiation complex (EDC) (see Fig. 1), a cluster of genes encoding proteins found in the uppermost layers of the epidermis, which are of great importance for keratinocyte differentiation and skin barrier maintenance [6]. Genes located in the EDC include loricrin $(L O R)$, involucrin $(I V L)$, filaggrin $(F L G)$, the small proline rich protein $(S P R R)$ genes and the late cornified envelope $(L C E)$ genes. This last cluster, with a total of 18 members, is divided into six families, LCE 1-6, based on related amino acid sequences, genomic organization and expression patterns [7]. The focus on genes involved in skin barrier function as susceptibility factors for atopic dermatitis and psoriasis is not entirely new. Studies on permeability, barrier

*Address correspondence to this author at the Department of Dermatology, Radboud University Nijmegen Medical Centre, P.O. Box 9101, 6500 HB Nijmegen, The Netherlands; Tel: +31 243614094; Fax: +31 243541184; E-mail: J.Schalkwijk@ncmls.ru.nl function and morphological examinations of patients' epidermis demonstrated an impaired barrier function for both diseases [8, 9]. Already in 1999, it was proposed that a genetic predisposition to a defective skin barrier was a primary event in the development of atopic dermatitis, allowing allergen penetration and enhanced Th2 responses [10].

\section{THE FILAGGRIN ATOPIC DERMATITIS CONNECT- ION: A CHANGE OF PARADIGM}

In atopic dermatitis, until recently regarded as a primarily immune-mediated condition, the disrupted barrier function of the skin is now considered as a major cause of the disease. Loss-of-function mutations in FLG were shown to be associated with atopic dermatitis in several populations [11, 12]. Large population based studies show a population attributable risk (PAR) of $13.5 \%$. As the $F L G$ gene is located in or near PSORS4, FLG mutations were also studied in psoriasis patients, however no associations were found [13].

Filaggrin is specifically expressed in the epidermis, where it has a function in the aggregation of keratin filaments, which is necessary for the formation of the stratum corneum [14]. Subsequently, within the stratum corneum, filaggrin is degraded into a pool of hydrophilic amino acids, which make up the natural moisturizing factor (NMF). These molecules play a central role in maintaining the hydration of the stratum corneum. NMF levels have been shown to correlate with FLG null allele status, and may therefore directly contribute to the dry skin phenotype seen in atopic dermatitis [15]. In addition to the structural properties of filaggrin, a filaggrin breakdown product, 


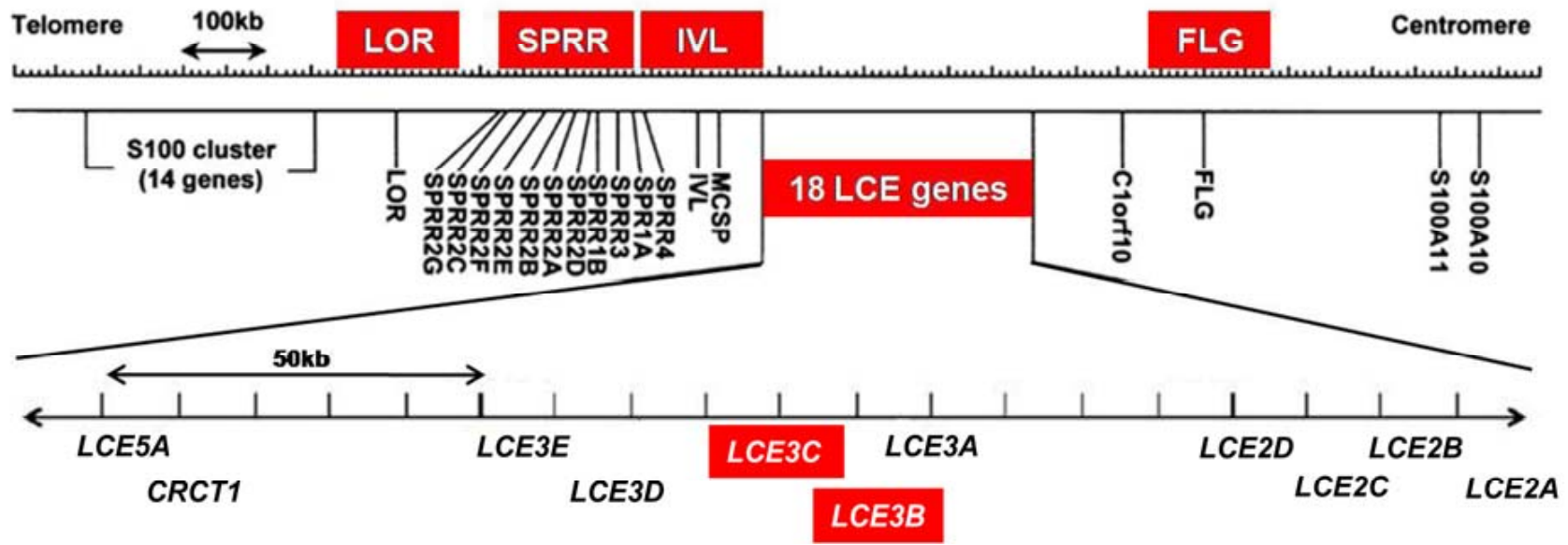

Fig. (1). Overview of the EDC region on chromosome 1. The EDC region is shown in the top half of the diagram, with the genes $L O R$, the SPRR family, IVL and FLG highlighted. Loss-of-function mutations in $F L G$ are associated with atopic dermatitis. In the lower half of the diagram a part of the LCE cluster, which consists of a total of 18 genes, is present. The deletion of $L C E 3 B$ and $L C E 3 C$ (highlighted) is associated with psoriasis. Adapted by permission from Macmillan Publishers Ltd: J Invest Dermatol [7], copyright 2005.

urocanic acid, is a UV-absorbing molecule [16] and the cisisomer has local and systemic immunosuppressive effects, which is demonstrated in murine models and in human keratinocytes and leukocytes in vitro [17].

Interestingly, also the cytokine environment associated with atopic dermatitis appears to contribute to compromised skin barrier function, as interleukin-4 and interleukin-13 suppress filaggrin gene expression [18]. Studies on flaky tail mice, harboring a mutation analogous to the common human FLG mutation, demonstrated that topical application of allergens resulted in cutaneous infiltration and an allergenspecific antibody response. These data demonstrate that antigen transfer through a defective epidermal barrier could be a key mechanism in atopic diseases [19].

\section{IDENTIFICATION OF THE $L C E 3 B / C$ DELETION AS THE PSORIASIS ASSOCIATED RISK FACTOR IN THE PSORS4 LOCUS}

The question if psoriasis is an epithelial disease or an immune-mediated disease has generated considerable debate over the last decades, but remains essentially unresolved. Until the early eighties psoriasis was considered to be a keratinocyte disease, but the advent of cyclosporin A as an effective mechanism-based treatment has changed this [20]. Many studies have even assumed an autoimmune basis of psoriasis. This was, however, largely founded on circumstantial evidence, as autoantibodies or autoreactive Tcells have never been demonstrated. For the last three decades psoriasis was primarily regarded as an immunemediated disease although this view is changing rapidly [21, 22]. Independent genetic studies have shown that PSORSI is the strongest and invariably reproduced psoriasis susceptibility locus. This locus contains several genes including $H L A-C w 6$, involved in adaptive immunity, and corneodesmosin $(C D S N)$, encoding a skin barrier protein expressed in differentiated keratinocytes. However, due to linkage disequilibrium (LD), the tendency of two genes to be inherited together more often than would be predicted by chance, it is difficult to identify the true causative susceptibility gene of the PSORS1 locus [23-25].
Although most of the available genetic and clinical data supported a role for T-cell driven processes, the importance of epidermis-expressed genes could not be ruled out completely. In addition to the genetic association with PSORS4 and the possible role of CDSN, cell biological studies implied that keratinocytes of psoriatic patients are intrinsically different from keratinocytes of healthy controls or atopic dermatitis patients. Cultured keratinocytes from patients were found to react differently to stimuli like cytokines, most likely due to genetically programmed differences [26, 27].

A hallmark of lesional psoriatic skin is premature keratinocyte differentiation and disturbed keratinization, including altered formation of the cornified envelope. In psoriatic skin the components of the cornified envelope are differentially expressed as compared to normal skin. The expression of the early differentiation markers like involucrin, corneodesmosin, the small proline-rich proteins, cystatin A and transglutaminase 1 is upregulated, while the expression of the late differentiation markers like loricrin and filaggrin is downregulated [28, 29]. The defective formation of the cornified envelope influences the barrier capacity of the skin in psoriasis patients. Several independent studies demonstrated that the transepidermal water loss (TEWL) of lesional psoriatic skin is higher compared to the TEWL of normal and uninvolved psoriatic skin. Furthermore, the level of the TEWL is related to the clinical severity of the lesion, suggesting that the barrier function of lesional psoriatic skin is weaker than in normal skin $[30,31]$. Another indication for the involvement of the physical barrier function of the skin is the Koebner phenomenon, i.e. the appearance of psoriatic lesions in uninvolved skin of psoriatic patients as a consequence of superficial trauma [32].

Until recently, no genetic association of specific EDC genes, e.g FLG and $L O R$, with psoriasis was found. De Cid et al. [33], however, demonstrated an association of the deletion of $L C E 3 B$ and $L C E 3 C$ with psoriasis in populations from Spain, Italy, The Netherlands and the USA. This 
common deletion was found with a higher frequency in psoriasis patients (68\%) compared to controls $(59 \%)$ with a corresponding PAR of $23 \%$. These results have been replicated in a German cohort [34]. In addition, in an independent genome wide association study of a large Chinese cohort, single nucleotide polymorphisms (SNPs) in strong $\mathrm{LD}$ with the $L C E 3 B / C$ deletion were identified as risk factors for psoriasis [35]. Interestingly, the $L C E 3 B / C$ deletion was not found to be associated with psoriatic arthritis [36].

In order to investigate the putative function of the LCE3B and LCE3C proteins, De Cid et al. [37] investigated the expression of the $L C E 3 C$ (located in the deletion, and therefore absent in some individuals) and $L C E 3 E$ genes (not located in the deletion and therefore used as a reference) in epidermal sheets of normal and psoriatic skin. The expression of $L C E 3 C$ and $L C E 3 E$ was hardly detectable in normal and uninvolved psoriatic skin. Though, in lesional psoriatic skin, both $L C E 3 C$ (when at least one intact $L C E 3 C$ allele was present) and $L C E 3 E$ were highly induced. To examine if skin barrier disruption might be a pathophysiological stimulus for $L C E 3$ expression, $L C E 3 C$ and $L C E 3 E$ expression was analysed in healthy individuals following minor skin injury by tape stripping. Indeed, in normal skin both genes were induced upon tape stripping, suggesting that induction of these genes is a repair response.

The $L C E$ genes have not been extensively studied. In mice, Marshall and colleagues [37] showed that members of the LCE family of proteins are incorporated into the cornified envelope via crosslinking by transglutaminases rather late in the process of epidermal differentiation, and it was proposed that these proteins modulate barrier activity on the skin surface. Jackson et al. [7] demonstrated that LCE1 and 2 family members are primarily expressed in skin, and that they are barely expressed in internal epithelia. The $L C E 3$ family members are variably expressed at low levels in epithelia, and they show varying expression between tissue types. LCE4 and LCE5 expression was hardly detected in the investigated tissues. LCE6 expression was not analysed. In cultured keratinocytes, Jackson et al. [7] revealed that the $L C E 2$ expression is induced by calcium, and UV induces the expression of the LCE1 and LCE2 families and of the $L C E 3 E$ gene.

\section{CONCLUDING REMARKS}

Genetic studies on inflammatory skin diseases, and on complex diseases in general, have proven to be useful tools to provide new insight into their pathophysiology. For atopic dermatitis, mutations found in $F L G$ have challenged the dogma that atopic dermatitis is primarily an immunemediated disease, and suggest skin barrier deficiency as a major cause. The association of the deletion of $L C E 3 B$ and $L C E 3 C$ with psoriasis is the first replicated susceptibility factor in the EDC, for this disease. We can only hypothesize about the role of the $L C E 3 B / C$ deletion in the pathogenesis of psoriasis. Since the expression of $L C E 3 C$ is induced after barrier disruption, we propose that incomplete barrier repair after minor injury might lead to penetration of environmental antigens or stimuli through the skin. These could trigger an inflammatory process that leads to psoriasis. Clearly, the molecular details of such a scenario need to be elucidated.
The nature of the environmental stimuli is at present unclear but microbial components e.g. from Streptococcus spp. would be excellent candidates. In general, components from the commensal flora or transient infection of throat and gut could very well trigger skin inflammation. The recently initiated human microbiome project is likely to contribute to novel insights in the pathogenesis of inflammatory skin diseases [38, 39]. Analysis of the skin microbiome in psoriasis patients and controls has already revealed differences in microbial community structure [40].

Altogether, these findings demonstrate that in addition to immune mechanisms, genetic variation of skin barrier genes contribute to major skin diseases such as atopic dermatitis and psoriasis.

ABBREVIATIONS
$\begin{aligned} & \text { CDSN } \\ & \text { EDC }\end{aligned}$ Corneodesmosin
FLG $=$ Fidermal differentiation complex
IVL $=$ Involucrin
LCE $=$ Late cornified envelope
LD $=$ Linkage disequilibrium
LOR $=$ Loricrin
NMF $=$ Natural moisturizing factor
PAR $=$ Population attributable risk
PSORS $=$ Psoriasis susceptibility locus
SNPs $=$ Single nucleotide polymorphisms
SPRR $=$ Small proline rich protein genes
TEWL $=$ Transepidermal water loss

\section{REFERENCES}

[1] Bieber T. Atopic dermatitis. N Engl J Med 2008; 358(14): 1483-94.

[2] Bowcock AM, Krueger JG. Getting under the skin: the immunogenetics of psoriasis. Nat Rev Immunol 2005; 5(9): 699711.

[3] Nestle FO, Kaplan DH, Barker J. Psoriasis. N Engl J Med 2009; 361(5): 496-509.

[4] Cookson W. The immunogenetics of asthma and eczema: a new focus on the epithelium. Nat Rev Immunol 2004; 4(12): 978-88.

[5] Elder JT, Bruce AT, Gudjonsson JE, et al. Molecular dissection of psoriasis: integrating genetics and biology. J Invest Dermatol 2009; [Epub ahead of print].

[6] Mischke D, Korge BP, Marenholz I, Volz A, Ziegler A. Genes encoding structural proteins of epidermal cornification and S100 calcium-binding proteins form a gene complex ("epidermal differentiation complex") on human chromosome 1q21. J Invest Dermatol 1996; 106(5): 989-92.

[7] Jackson B, Tilli CM, Hardman MJ, et al. Late cornified envelope family in differentiating epithelia--response to calcium and ultraviolet irradiation. J Invest Dermatol 2005; 124(5): 1062-70.

[8] Cork MJ, Robinson DA, Vasilopoulos Y, et al. New perspectives on epidermal barrier dysfunction in atopic dermatitis: geneenvironment interactions. J Allergy Clin Immunol 2006; 118(1): 321.

[9] Proksch E, Brandner JM, Jensen JM. The skin: an indispensable barrier. Exp Dermatol 2008; 17(12): 1063-72.

[10] Taieb A. Hypothesis: from epidermal barrier dysfunction to atopic disorders. Contact Dermatitis 1999; 41(4): 177-80.

[11] Palmer CN, Irvine AD, Terron-Kwiatkowski A, et al. Common loss-of-function variants of the epidermal barrier protein filaggrin are a major predisposing factor for atopic dermatitis. Nat Genet 2006; 38(4): 441-6. 
[12] Rodriguez E, Baurecht H, Herberich E, et al. Meta-analysis of filaggrin polymorphisms in eczema and asthma: robust risk factors in atopic disease. J Allergy Clin Immunol 2009; 123(6): 1361-70.

[13] Huffmeier U, Traupe H, Oji V, et al. Loss-of-function variants of the filaggrin gene are not major susceptibility factors for psoriasis vulgaris or psoriatic arthritis in German patients. J Invest Dermatol 2007; 127(6): 1367-70.

[14] Candi E, Schmidt R, Melino G. The cornified envelope: A model of cell death in the skin. Nat Rev Mol Cell Biol 2005; 6(4): 328-40.

[15] Kezic S, Kemperman PM, Koster ES, et al. Loss-of-function mutations in the filaggrin gene lead to reduced level of natural moisturizing factor in the stratum corneum. J Invest Dermatol 2008; 128(8): 2117-9.

[16] De Fine OF, Wulf HC, Therkildsen P, Poulsen T, Crosby J, Norval M. Urocanic acid isomers: relation to body site, pigmentation, stratum corneum thickness and photosensitivity. Arch Dermatol Res 1997; 289(9): 501-5.

[17] Brown SJ, McLean WH. Eczema genetics: current state of knowledge and future goals. J Invest Dermatol 2009; 129(3): 54352.

[18] Howell MD, Kim BE, Gao P, et al. Cytokine modulation of atopic dermatitis filaggrin skin expression. J Allergy Clin Immunol 2007; 120(1): 150-5.

[19] Fallon PG, Sasaki T, Sandilands A, et al. A homozygous frameshift mutation in the mouse Flg gene facilitates enhanced percutaneous allergen priming. Nat Genet 2009; 41(5): 602-8.

[20] Ellis CN, Gorsulowsky DC, Hamilton TA, et al. Cyclosporine improves psoriasis in a double-blind study. JAMA 1986; 256(22): 3110-6.

[21] Nickoloff BJ. Keratinocytes regain momentum as instigators of cutaneous inflammation. Trends Mol Med 2006; 12(3): 102-6.

[22] Hollox EJ, Huffmeier U, Zeeuwen PL, et al. Psoriasis is associated with increased beta-defensin genomic copy number. Nat Genet 2008; 40(1): 23-5.

[23] Capon F, Trembath RC, Barker JN. An update on the genetics of psoriasis. Dermatol Clin 2004; 22(4): 339-47, vii.

[24] Nair RP, Stuart P, Henseler T, et al. Localization of psoriasissusceptibility locus PSORS1 to a $60-\mathrm{kb}$ interval telomeric to HLAC. Am J Hum Genet 2000; 66(6): 1833-44.

[25] Orru S, Giuressi E, Carcassi C, Casula M, Contu L. Mapping of the major psoriasis-susceptibility locus (PSORS1) in a 70-Kb interval around the corneodesmosin gene (CDSN). Am J Hum Genet 2005; 76(1): 164-71.

[26] Giustizieri ML, Mascia F, Frezzolini A, et al. Keratinocytes from patients with atopic dermatitis and psoriasis show a distinct chemokine production profile in response to $\mathrm{T}$ cell-derived cytokines. J Allergy Clin Immunol 2001; 107(5): 871-7.
Zeeuwen PL, de Jongh GJ, Rodijk-Olthuis D, et al. Genetically programmed differences in epidermal host defense between psoriasis and atopic dermatitis patients. PLoS One 2008; 3(6): e2301.

[28] Ishida-Yamamoto A, Takahashi H, Iizuka H. Immunoelectron microscopy links molecules and morphology in the studies of keratinization. Eur J Dermatol 2000; 10(6): 429-35.

[29] Iizuka H, Takahashi H, Honma M, Ishida-Yamamoto A. Unique keratinization process in psoriasis: late differentiation markers are abolished because of the premature cell death. J Dermatol 2004; 31(4): 271-6.

[30] Grice K, Sattar H, Baker H, Sharratt M. The relationship of transepidermal water loss to skin temperature in psoriasis and eczema. J Invest Dermatol 1975; 64(5): 313-5.

[31] Motta S, Monti M, Sesana S, Mellesi L, Ghidoni R, Caputo R. Abnormality of water barrier function in psoriasis. Role of ceramide fractions. Arch Dermatol 1994; 130(4): 452-6.

[32] Powles AV, Baker BS, Rutman AJ, McFadden JP, Valdimarsson $\mathrm{H}$, Fry L. Epidermal rupture is the initiating factor for the Koebner response in psoriasis. Acta Derm Venereol 1990; 70(1): 35-8.

[33] de Cid R, Riveira-Munoz E, Zeeuwen PL, et al. Deletion of the late cornified envelope LCE3B and LCE3C genes as a susceptibility factor for psoriasis. Nat Genet 2009; 41(2): 211-5.

[34] Huffmeier U, Bergboer JGM, Becker T, et al. Replication of $L C E 3 C-L C E 3 B \mathrm{CNV}$ as a risk factor for psoriasis. Analysis of interaction with other genetic risk factors. J Invest Dermatol 2009; [Epub ahead of print].

[35] Zhang XJ, Huang W, Yang S, et al. Psoriasis genome-wide association study identifies susceptibility variants within LCE gene cluster at 1q21. Nat Genet 2009; 41(2): 205-10.

[36] Huffmeier U, Estivill X, Riveira-Munoz E, et al. Deletion of LCE3C and LCE3B genes at PSORS4 does not contribute to Susceptibility to Psoriatic Arthritis in German patients. Ann Rheum Dis 2009; [Epub ahead of print].

[37] Marshall D, Hardman MJ, Nield KM, Byrne C. Differentially expressed late constituents of the epidermal cornified envelope. Proc Natl Acad Sci USA 2001; 98(23): 13031-6.

[38] Grice EA, Kong HH, Conlan S, et al. Topographical and temporal diversity of the human skin microbiome. Science 2009; 324(5931): 1190-2.

[39] Turnbaugh PJ, Ley RE, Hamady M, Fraser-Liggett CM, Knight R, Gordon JI. The human microbiome project. Nature 2007; 449(7164): 804-10.

[40] Gao Z, Tseng CH, Strober BE, Pei Z, Blaser MJ. Substantial alterations of the cutaneous bacterial biota in psoriatic lesions. PLoS One 2008; 3(7): e2719.

(C) Bergboer et al.; Licensee Bentham Open.

This is an open access article licensed under the terms of the Creative Commons Attribution Non-Commercial License (http://creativecommons.org/licenses/by-nc/ 3.0/) which permits unrestricted, non-commercial use, distribution and reproduction in any medium, provided the work is properly cited. 